\begin{tabular}{c} 
International Journal of Engineering \&Technology, $7(1.8)(2018) 178-181$ \\
SPC \\
International Journal of Engineering \& Technology \\
Website: $w w w$. sciencepubco.com/index.php/IJET \\
Research paper \\
\hline
\end{tabular}

\title{
Consensus Based Economic Dispatch including System Power Losses
}

\author{
K. Naga Tejaswini ${ }^{1 *}$, G. Kesava Rao ${ }^{2}$ \\ ${ }^{1}$ M.Tech Student, Department of Electrical \& Electronics Engineering, Koneru Lakshmaiah Educational Foundation, \\ Vaddeswaram, Guntur, Andhra Pradesh, India \\ ${ }^{2}$ Professor, Department of Electrical \& Electronics Engineering, Koneru Lakshmaiah Educational Foundation, \\ Vaddeswaram, Guntur, Andhra Pradesh, India \\ *E-mail:tejaswini.chinni17@gmail.com
}

\begin{abstract}
Economic dispatch (ED) is an important class of optimization problem in Power System Operation. As both conventional and heuristic methods to solve EDP are centrally controlled, which may leads to some performance limitations, a Consensus based distributed algorithm is proposed in this paper to solve Economic Dispatch with inclusion of losses. Earlier, some papers dealt with the consensus based methods to solve Economic dispatch, but here in this paper the losses are included and the variation of losses at each iteration are also used to update the mismatch, which has some major prominence in the present day Power system environment. In this paper, the mismatch between load demand and total power generation is collectively learnt by the each generator, unlike the centralized approach, through the strongly connected communication network. MATLAB results in IEEE 6-bus system validate the potency and efficacy of the proposed technique
\end{abstract}

Keywords: Consensus-based methods; Decentralization; Economic dispatch; Transmission loss

\section{Introduction}

With the day-to-day increase in Power demand, there is a need to equip impending Power Systems with large number of Distributed Energy Resources (DERs), like DGs, energy storage devices (like batteries) and most advanced Communication networks, which lead to Deregulated Restructured Power system operation, with the Consumer participation in the Electricity Market. Some of the cardinal challenges in Power Systems (especially in distribution), like Economic dispatch are revisited with the help of transpiring Smart grid structure. Economic Dispatch is a fundamental problem, which is generally formulated as an optimization problem with the objective to reduce the total cost of generation, subject to several constraints (like Power Balance, Power limits and others). Along with traditional optimization techniques like $\lambda$ - iteration method and linear programming, some of the heuristic methods like Genetic Algorithm (GA) [4] and Particle Swarm Optimization (PSO) [5] are also employed to solve EDP. All these methods are of centrally controlled. For secure operation, decentralized operation is preferred over the centralized one.

Consensus based distributed methods have been found to be feasible in many Multi-Agent System applications, such as in computer networks, Industrial, commercial and automated systems. The major concern in a consensus problem is to achieve an accord regarding concern quantities associated with agents in the MultiAgent Systems through the local information exchange. Recently, researchers are interested towards the application of consensus methods to smart grid related problems.

In the literature, ED is well-documented; [6] proposes Consensus approach, [7] proposes bisection approach, [8] proposes a designing scheme for EDP, $[9,10]$ proposes the distributed approach, $[11,12]$ proposes the advanced applications of consensus in power system operation.

In this paper, a distributed solution is furnished to solve the EDP with the inclusion of losses and here the losses are not taken as constant and the losses are updated and included at each iteration of the proposed Consensus algorithm.

\section{Consensus Methods}

\subsection{Problem Formulation:}

The Economic dispatch problem is formulated as follows:

$\operatorname{Min} . C_{i}\left(P_{G i}\right)=\sum_{i=1}^{N_{g}}\left(a_{i} P_{G i}^{2}+b_{i} P_{G i}+c_{i}\right)$

Subject to: (i) System power balance

$P_{D}+P_{L}-\sum_{i=1}^{N_{g}} P_{G i}=0$

Where, $P_{D}$ is the total system load demand $P_{L}$ is the system power loss

$P_{G i}$ is the power generated at the $\mathrm{i}^{\text {th }}$ generator $\left(\mathrm{i}=1,2, \ldots \ldots, \mathrm{N}_{\mathrm{g}}\right)$

(ii) $P_{G i}^{\min } \leq P_{G i} \leq P_{G i}^{\max }$

The above problem, a Lagrangian unconstrained objective function is formulated and is minimized using gradient methods. The well known solution of the above problem $[1,2,3]$ is given below: 
(i) If the power losses are neglected,

$$
(\mathrm{IC})_{1}=(\mathrm{IC})_{2}=\ldots=(\mathrm{IC})_{\mathrm{Ng}}=\lambda
$$

Where, (IC) $)_{\mathrm{i}}$ is the Incremental Cost at the $\mathrm{i}^{\text {th }}$ generator.

$$
(I C)_{i}=2 a_{i} P_{G i}+b_{i}=\lambda
$$

(ii) If the losses are taken into account, then

$$
\lambda=\frac{(I C)_{i}}{1+(I T L)_{i}}
$$

Where, (ITL) is the Incremental Transmission Loss defined as

$(I T L)_{i}=\frac{\partial P_{L}}{\partial P_{G i}}$

$\lambda$ is called the Lagrange Multiplier and indicates the cost of generation.

The eqs. (4) \& (5) are solved by the well-known lambda iteration method.

\subsection{Consensus - based methods:}

Let the system be operating in steady state with a specified load and power injections from the generators. Thus, each generator has its own $\lambda_{i}=2 a_{i} P_{G i}+b_{i}$ and at the optimum point as indicated by eq. (4), all lambdas must converge to a single value; that is the generator powers reach a consensus. Unlike the traditional $\lambda$ - iteration solution which is totally centralized, the consensus algorithm can be distributed and can be implemented with only the loca data. Because of the problems posed by the centralized methods (like demanding high bandwidth for communication network and also the sensitivity of the power network even for minute faults in communication network), the system operators prefer to go for decentralized or distributed algorithms. The consensus algorithm is one of such methods and easy to implement and robust in nature.

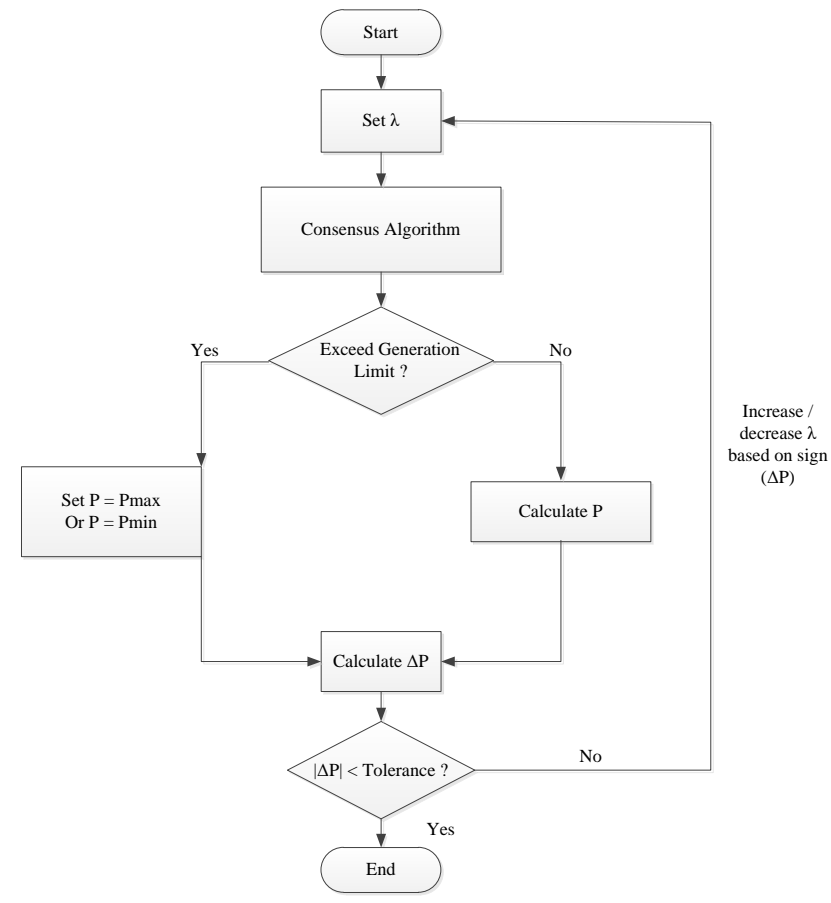

Fig. 1. Flowchart for Incremental Cost Consensus (ICC) Algorithm Fig. 1 shows the flowchart for the Incremental Cost Consensus (ICC) Algorithm.

\subsection{Graph Theory:}

The power system nodes and the lines can be represented by a graph, which is necessary in designing a communication network for the power system. Let the graph be defined as $G=(V, E$, A) where vertex $V$ is a set of power system nodes, $E$ is a set of pairs of nodes called edges and $\mathrm{A}$ is a real matrix of dimension $\mathrm{m} \times \mathrm{m}$ and is called an Adjacency matrix; its $\mathrm{ij}^{\text {th }}$ element represents distance between nodes $\mathrm{i}$ and $\mathrm{j}$. A node, $\mathrm{i}$ can have a set of neighbors and this set is indicated by $\mathrm{N}_{\mathrm{i}}$. The number of edges between nodes $\mathrm{i}$ and $\mathrm{j}$ is called the distance between the nodes. The greatest minimum distance between the nodes is called the diameter of the network. This diameter is an important parameter to convert the locally available data to global data in a distributed control.

\subsection{The Row - Stochastic Matrix:}

Given the matrix A, the adjacency matrix, as described above, a matrix called Laplacian matrix can be formulated as [15].

$$
\begin{aligned}
& l_{i j}=\sum_{i \neq j} a_{i j} \text { for diagonal elements } \\
& l_{i j}=-a_{i j} \text { for off-diagonal elements }
\end{aligned}
$$

The Laplacian matrix gives considerable information on the connectivity of the graph, which is a measure of the convergence speed [11]. From the Laplacian matrix, the Row - Stochastic Matrix D, can be derived as

$$
d_{i j}=\frac{\left|l_{i j}\right|}{\sum_{j=1}^{n}\left|l_{i j}\right|} ; \quad \mathrm{i}=1,2, \ldots . ., \mathrm{n}
$$

This matrix D is square, has elements all non - negative and real; also the sum of each row adds to unity.

The matrix $\mathrm{D}$ for a five node communication network can be derived as follows:

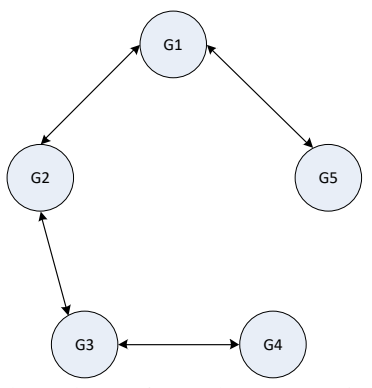

Fig. 2. Typical topology of 5-node Communication network

The Row Stochastic Matrix for the given 5-node Communication network in Fig. 2 is

$$
\mathrm{D}=\left[\begin{array}{lllll}
0.5 & 0.25 & 0 & 0 & 0.25 \\
0.25 & 0.5 & 0.25 & 0 & 0 \\
0 & 0.25 & 0.5 & 0.25 & 0 \\
0 & 0 & 0.5 & 0.5 & 0 \\
0.5 & 0 & 0 & 0 & 0.5
\end{array}\right]
$$

In the same way, as the communication topology differs, the Row stochastic matrix also differs accordingly. Thus, the Convergence of the consensus algorithm also depends on the communication network topology.

The $\mathrm{D}$ - matrix plays a pivotal role in bringing the state of the neighboring nodes to consensus, as given in the following sections.

\subsection{First-Order Consensus:}

The discrete time consensus algorithm is given by 


$$
x_{i}(k+1)=x_{i}(k)+u_{i}(k)
$$

Where, $x_{i}(k)$ and $u_{i}(k)$ are the state and control inputs at node $i$ at time interval $\mathrm{K}$.

In the decentralized local control,

$$
u_{i}(k)=\sum_{j \in N_{i}}\left(x_{j}(k)-x_{i}(k)\right)
$$

When the consensus is reached, $u_{i}(k)=0$; this and the fact that $\sum_{j \in N_{i}} d_{i j}=1$ give

$$
x_{i}(k+1)=\sum_{j \in N_{i}} d_{i j} x_{j}(k)
$$

In the ED, the state variable is the multiplier, $\lambda$. When the load demand changes, the $\lambda$ and the generator injections too will change;

Hence

$$
\lambda_{i}(k+1)=\sum_{j \in N_{i}} d_{i j} \lambda_{j}(k)+\Delta \lambda_{i}
$$

Here $\Delta \lambda_{i}$ represents any changes in the state of the system following a change in the power demand. So,

$$
\begin{gathered}
\lambda_{i}=\lambda_{i}\left(P_{i}\right) ; \quad \Delta \lambda_{i}=\frac{\partial \lambda_{i}}{\partial P_{G i}} \Delta P_{G i} \\
\lambda_{i}=2 a_{i} P_{G i}+b_{i} \\
\Delta \lambda_{i}=2 a_{i} \Delta P_{G i}
\end{gathered}
$$

So, $\Delta \lambda_{i}$ is proportional to $\Delta P_{G i}$

$$
\lambda_{\mathrm{i}}(\mathrm{k}+1)=\sum_{\substack{\mathrm{j} \in \mathrm{N}_{\mathrm{i}} \\ \mathrm{S}_{\mathrm{G}}}} \mathrm{d}_{\mathrm{ij}} \lambda_{\mathrm{i}}(\mathrm{k})+\mathrm{K}_{\mathrm{p}} 2 \mathrm{a}_{\mathrm{i}} \Delta \mathrm{P}_{\mathrm{i}} ; \forall \mathrm{i} \in \mathrm{S}_{\mathrm{G}}
$$

In order to make the Consensus to occur faster, the second term on the right hand side of the above $\lambda(\mathrm{k}+1)$ equation be changed to $2 a_{i} \Delta P_{i}(k)$, where $\Delta P_{i}(k)$ is the global power mismatch including the system losses as seen from node $\mathrm{i}$

$$
\Delta P_{i}(k)=\left(P_{D}+P_{L}\right)-\sum_{i=1}^{N_{g}} P_{G i}
$$

The lambda multiplier is computed for each of generator buses $(\mathrm{i}=$ $1,2, \ldots \ldots, \mathrm{N}_{\mathrm{g}}$ ) at every iteration. The consensus will be reached fast because the control parameter used with each $\lambda_{i}$ is the global mismatch, Load flow is run offline at the end of each Consensus algorithm, so as to update $\lambda$ value.

\section{The Algorithm:}

The algorithm for the proposed method is as follows:

Step 1: Read initial data: $P_{G i}\left(\mathrm{i}=1,2, \ldots, \mathrm{N}_{\mathrm{g}}\right), P_{D}, \lambda_{i}(\mathrm{i}=1,2, \ldots$. $\mathrm{Ng}_{\mathrm{g}}$, Tolerance $\left(10^{-4}\right)$, Row Stochastic Matrix.

Step 2: Let there be an increase in demand at the load nodes, resulting in power mismatch at each node and hence at global level.

Step 3: Update the lambda values at generator node as per eqs. (11) $\&(14)$.

Step 4: Compute the generator powers with the lambdas obtained in step 3.

Step 5: Run the load flow offline with the $P_{G i}\left(\mathrm{i}=1,2, \ldots, \mathrm{N}_{\mathrm{g}}\right)$ and the specified $P_{D}$, obtain the system losses.

Step 6: Compute the system power mismatch $\Delta P=\left(P_{D}+P_{L}-\sum P_{G i}\right)$
Step 7: Go to step 3, repeat the same until all the lamdas at generator nodes reach a unique value.

\section{Results of Simulation:}

The proposed methodology has been used on IEEE 6-bus system, with 3 generator nodes with quadratic cost curves [1]. The consensus algorithm is implemented with the changes in the power demand at the load buses. This change is used in the second term on the right hand side of eq.(16) [16]. The literature does not show the consensus-based algorithms with updation of losses. The proposed algorithm takes into account the variation in the losses also in obtaining the local power mismatch. The losses and the change in the losses at each iteration have been computed by off- line power flow; the line flows at each node, the losses are computed locally and added to get global power mismatch, which is available at each generator node through the communication network and the information transfer.

Initially taking a system and considering the previous state of operation, and taking into consideration some change in load demand ( here it is 12\%), the lambdas of each generator is taken based on the powers from previous states. From there, the power flow is run offline and the losses and optimal powers are obtained from it. From there, the consensus algorithm is applied to the system by taking the optimal values from power flow. The power flow, which is run offline, helps in finding the line flows through which losses are obtained and thus Global power mismatch is obtained from the eq. (16). After finding the value of $\Delta P_{i}$ at each generator node, the lambda value is updated using the eq. (15). After finding the new values of lambdas, the respective generations are obtained. This iterative process is continued till all the lambda values reach a unique consensus value of lambda, which is the final optimal lambda for the given system, for the respective change in load demand.

Thus, at the end of each iteration of the consensus algorithm, the system power flow is run in order to obtain the system losses, from which the global power mismatch is calculated.

The results of lambda updating are shown in Table, where it can be seen that the initial lambdas converged to a unique consensus value in 16 iterations. The convergence analysis of lambdas are shown in the graphs.

Table: Consensus Lambda Update for $12 \%$ change in the load demand using proposed algorithm

\begin{tabular}{|l|c|c|c|l|}
\hline $\begin{array}{l}\text { Iteration } \\
\text { no. }\end{array}$ & $\boldsymbol{\lambda}_{\mathbf{1}}$ & $\boldsymbol{\lambda}_{\mathbf{2}}$ & $\boldsymbol{\lambda}_{\mathbf{3}}$ & $\begin{array}{l}\text { Total } \\
\text { Cost }\end{array}$ \\
\hline 1 & 12.8190 & 11.2220 & 11.7222 & 3189.5 \\
\hline 2 & 12.6670 & 11.7463 & 11.8713 & 3465.1 \\
\hline 3 & 12.3848 & 12.0077 & 12.0390 & 3443.4 \\
\hline 4 & 12.2669 & 12.1098 & 12.1176 & 3440.4 \\
\hline 5 & 12.2170 & 12.1510 & 12.1530 & 3440.2 \\
\hline 6 & 12.1958 & 12.1680 & 12.1685 & 3440.3 \\
\hline 7 & 12.1868 & 12.1751 & 12.1752 & 3440.4 \\
\hline 8 & 12.1830 & 12.1781 & 12.1781 & 3440.4 \\
\hline 9 & 12.1814 & 12.1793 & 12.1793 & 3440.4 \\
\hline 10 & 12.1807 & 12.1798 & 12.1798 & 3440.5 \\
\hline 11 & 12.1804 & 12.1801 & 12.1801 & 3440.5 \\
\hline 12 & 12.1803 & 12.1801 & 12.1801 & 3440.5 \\
\hline 13 & 12.1803 & 12.1802 & 12.1802 & 3440.5 \\
\hline 14 & 12.1802 & 12.1802 & 12.1802 & 3440.5 \\
\hline 15 & 12.1802 & 12.1802 & 12.1802 & 3440.5 \\
\hline 16 & 12.1802 & 12.1802 & 12.1802 & 3440.5 \\
\hline
\end{tabular}


The convergence details of the Consensus algorithm is shown in the following figures.

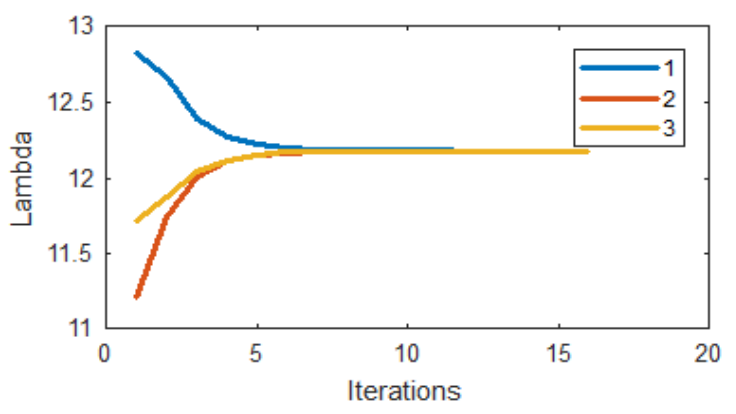

Fig. 3. Convergence of Lambda

Fig. 3 shows the convergence of lambdas to a unique consensus value. Starting from the individual values of lambda of each generator, a unique consensus value is reached by all the lambdas at the end of final iteration of the proposed consensus algorithm for a given change in load demand.

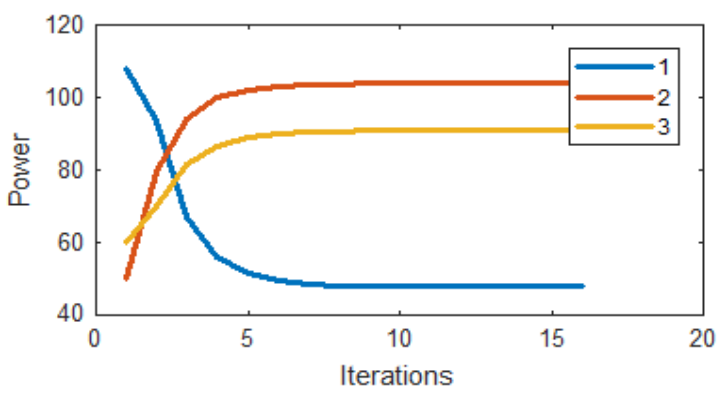

Fig. 4. Variation in the Power Generation

Fig. 4 shows the respective Powers generated at each iteration. Initially the power generations are taken from the previous states. And by the proposed consensus algorithm for a given change in the load demand, optimal power generations are obtained.

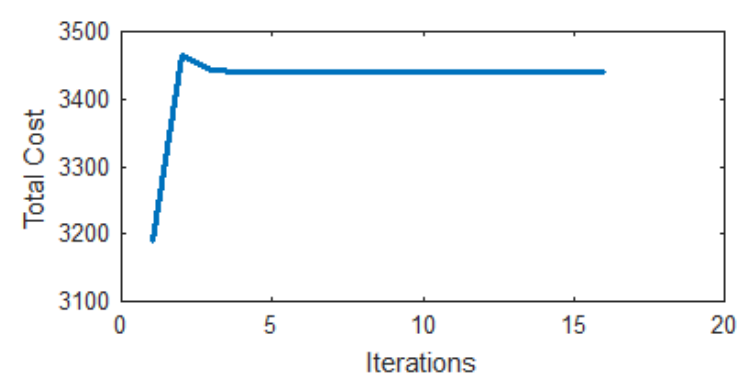

Fig. 5. Cost Convergence

Fig. 5 shows the Cost Vs Iterations characteristic. Here the initial cost is shown for the previously operating state of the system and later, for the given change in demand, the cost increases and from there it reaches to an optimal value by the proposed algorithm.

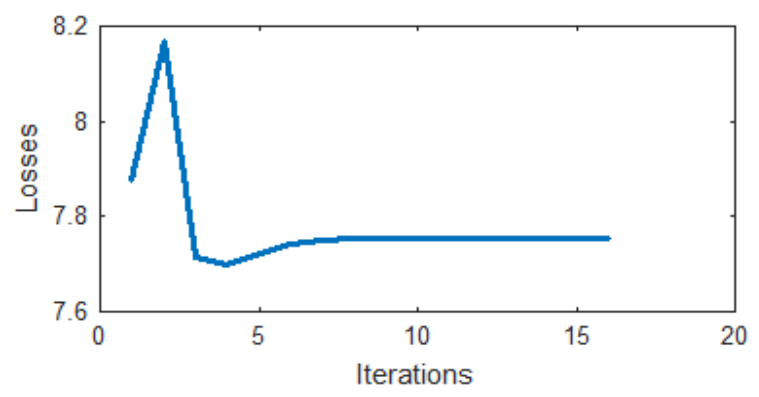

Fig. 6. Variation in Losses
Fig. 6 shows the respective losses at each iterations. From the offline power flow, the losses are obtained at each iteration of the proposed algorithm. Initially the losses shown will be for the previous state of operation and from there, for the change in the load demand, the losses will increase and from there it reaches an optimal value by the end of the consensus algorithm.

\section{Conclusion}

Consensus based Distributed solution for the Economic Dispatch Problem, with inclusion of losses based on the most up-to-date information is given in this paper. The proposed method relies on the strong communication network, handles the power mismatches locally, and determines the Global power mismatch. The efficacy of the presented method is validated with MATLAB - MATPOWER programming Simulations on IEEE 6-bus system.

\section{References}

[1] A. Bakirtzis, V. Petridis, S. Kazarlis, "Genetic Algorithm solution to the Economic dispatch", IEE Proc.-Gener. Transm. Distrib, Vol. 141 No. 4; pp.377-382, 1994.

[2] Zwe-Lee Gaing, "Particle Swarm Optimization to solving the Economic dispatch considering the generator constraints", IEEE Trans. Power Systems, Vol.18 No. 3, pp. 1187-1195, Aug. 2003.

[3] R. Wei, R. W. Beard, and E. M. Atkins, "A survey of consensus problems in multi-agent coordination", Proc. 2005 American Control Conf. , vol. 3, pp. 1859-1864, 2005.

[4] F Reza Olfati Saber, J Alex Fax, Richard M Murray, "Consensus and cooperation in networked Multi-Agent Systems", Proc IEEE, Vol. 95, No. 1, pp. 215-233, Jan. 2007.

[5] Shiping Yang, Sicong Tan, Jian - Xin Xu, "Consensus based approach for Economic Dispatch problem in Smart Grid", IEEE Trans. on Power Systems, Vol. 28, No.4, pp. 4416-4426, Nov 2013.

[6] Hao Xing, Yuting Mou, Minyue Fu, Zhiyun Lin, "Consensus based Bisection approach for Economic Power dispatch", 53 ${ }^{\text {rd }}$ IEEE Conf. on Decision and Control, Los Angeles, California, USA, Dec. 2014.

[7] Guanghui Wen and Wenwu Yu, Xinghuo Yu, Jinde Cao, "Designing adaptive Consensus based scheme for Economic Dispatch of Smart Grid", IEEE International Conference on Advanced Computational Intelligence, Thailand, Feb. 2016

[8] Xiaoshun Zhang, Hao Xu, Tao Yu, Bo Yang, Maoxin Xu, "Robust collaborative consensus algorithm for decentralized economic dispatch with a practical communiation network", Electric Power Systems Research Vol. 140, pp. 597-610, 2016.

[9] Yinliang $\mathrm{Xu}$ and Zhicheng Li, "Distributed Optimal resource management based on consensus algorithm in a microgrid", IEEE Trans. on Industrial Electronics, Vol. 62, No. 4, pp. 2584-2592, Apr. 2015.

[10] Wei Liu, Wei Gu, Yinliang Xu, Shuai Xue, Ming Chen, Bo Zhao, Miao Fan, "Improved average consensus algorithm based distributed cost optimization for load shedding of autonomous microgrids", Electrical Power and Energy Systems, Vol. 73, pp. 89-96, 2015.

[11] Ziang Zhang, Mo-Yuen Chow, "Convergence analysis of the Incremental Cost Consensus algorithm under different communication network topologies in a smart grid", IEEE Trans on Power Systems, Vol. 27, No. 4, pp. 1761-1768, Nov. 2012.

[12] A. J. Wood and B. F. Wollenberg, "Power Generation, Operation, and Control", New York, NY, USA: Wiley, 1996.

[13] John J Grainger, William D. Stevenson Jr, "Power System Analysis", 1994.

[14] Hadi Saadat, "Power System Analysis", 1999.

[15] Mo-Yuen Chow and Ziang Zhang, "Incremental cost consensus algorithm in a smart grid environment", Proc. IEEE Power \& Energy SocietyGeneral Meeting, Detroit,MI, 2011.

[16] Giulio Binetti, Ali Davoudi, Frank L. Lewis, David Naso, Biagio Turchiano, "Distributed consensus based economic dispatch with transmission losses", IEEE Trans Power Systems, Vol. 29, No. 4, pp. 1711-1720, July 2014. 